\title{
The effects of neuromuscular electrical stimulation for dysphagia in opercular syndrome: a case study
}

\author{
Laura W. J. Baijens · Renée Speyer • \\ Nel Roodenburg $\cdot$ Johannes J. Manni
}

Received: 1 April 2007 / Accepted: 19 October 2007 / Published online: 8 January 2008

(C) The Author(s) 2008

\begin{abstract}
A 76-year-old man with opercular syndrome characterized by complete bilateral loss of voluntary control of facial, lingual, pharyngeal and masticatory muscles is presented with focus on the severe dysphagia. Three years earlier the patient had experienced two strokes resulting in opercular syndrome with severe dysphagia. Despite initial logopedic dysphagia treatment, swallowing did not improve. A new treatment for dysphagia, consisting of neuromuscular electrical stimulation was applied on the patient. He returned to oral feeding. Clinical and treatment observations are reported.
\end{abstract}

Keywords Neuromuscular electrical stimulation . Opercular syndrome · Foix-Chavany-Marie syndrome · Dysphagia $\cdot$ Facio-glosso-pharyngo-masticatory paralysis

\section{Introduction}

Although Magnus was the first to describe the opercular syndrome in 1837 [18], the syndrome is also known as Foix-Chavany-Marie syndrome named after the French authors who described the syndrome in 1926 [9]. The classical opercular syndrome presents with loss of voluntary facial, pharyngeal, lingual, and mastication movements.

L. W. J. Baijens $(\bowtie) \cdot$ R. Speyer · J. J. Manni

Department of Otorhinolaryngology,

Head and Neck Surgery, University Hospital Maastricht, PO Box 5800, 6202, AZ Maastricht, The Netherlands

e-mail: LBA@skno.azm.nl

N. Roodenburg

Department of Neurology,

University Hospital Maastricht, Maastricht, The Netherlands
Reflexive and automatic movements with the exception of the gag reflex, are being preserved [13]. Usually the gag reflex is decreased or absent [1,13,22]. Automato-voluntary dissociation of movements with the muscles of the larynx, pharynx, tongue, lips, and cheeks has been described in oral apraxia as well $[1,8]$. The prevalence of the opercular syndrome is not known. Bilateral abnormality of the opercular cortex forms the neuroanatomical basis of the opercular syndrome in the classical cases [1]. Multiple strokes form the major etiology of this syndrome [13, 23]. The voluntary phase of the swallowing act is severely affected in the majority of these patients while reflex swallowing is preserved $[1,13,22]$. Very often, patients with opercular syndrome receive percutaneous endoscopic gastrostomy (PEG) tube feeding. In this case study, a new treatment for dysphagia consisting of neuromuscular electrical stimulation applied on a patient diagnosed with opercular syndrome, is reported $[10,17]$.

\section{Report of a case}

Three years ago, a 76-year-old male was referred to the Department of Neurology because of the sudden onset of difficulty to speak and weakness of the right arm. A left hemispheric infarction was diagnosed. Six months later he experienced a second stroke with inability to speak or swallow and a paralysis of the left arm. During neurologic examination no voluntary movements of the jaw, lips or tongue were present. A bilateral dysfunction of the 5 th, 7 th, 9th, 10th, and 12th cranial nerves was found with the exception of the automatic movements of the same muscles. These automatic movements were preserved. The gag reflex was decreased. Although the presence of the swallowing reflex was clinically observed, the patient totally 
depended on nasogastric tube feeding because of massive aspiration, including his own saliva. Besides the earlier leftsided cortical infarction, the MRI-scan showed diffuse atrophy of the brain. An infarction was diagnosed in the right pre- and post-central convolutions. Also, right-sided sub- cortical lacunar foci were seen (Fig. 1). The opercular syndrome was diagnosed.

Despite initial logopedic dysphagia treatment during the first year after the second stroke, the dysphagia did not improve [6] and the patient fully depended on PEG tube
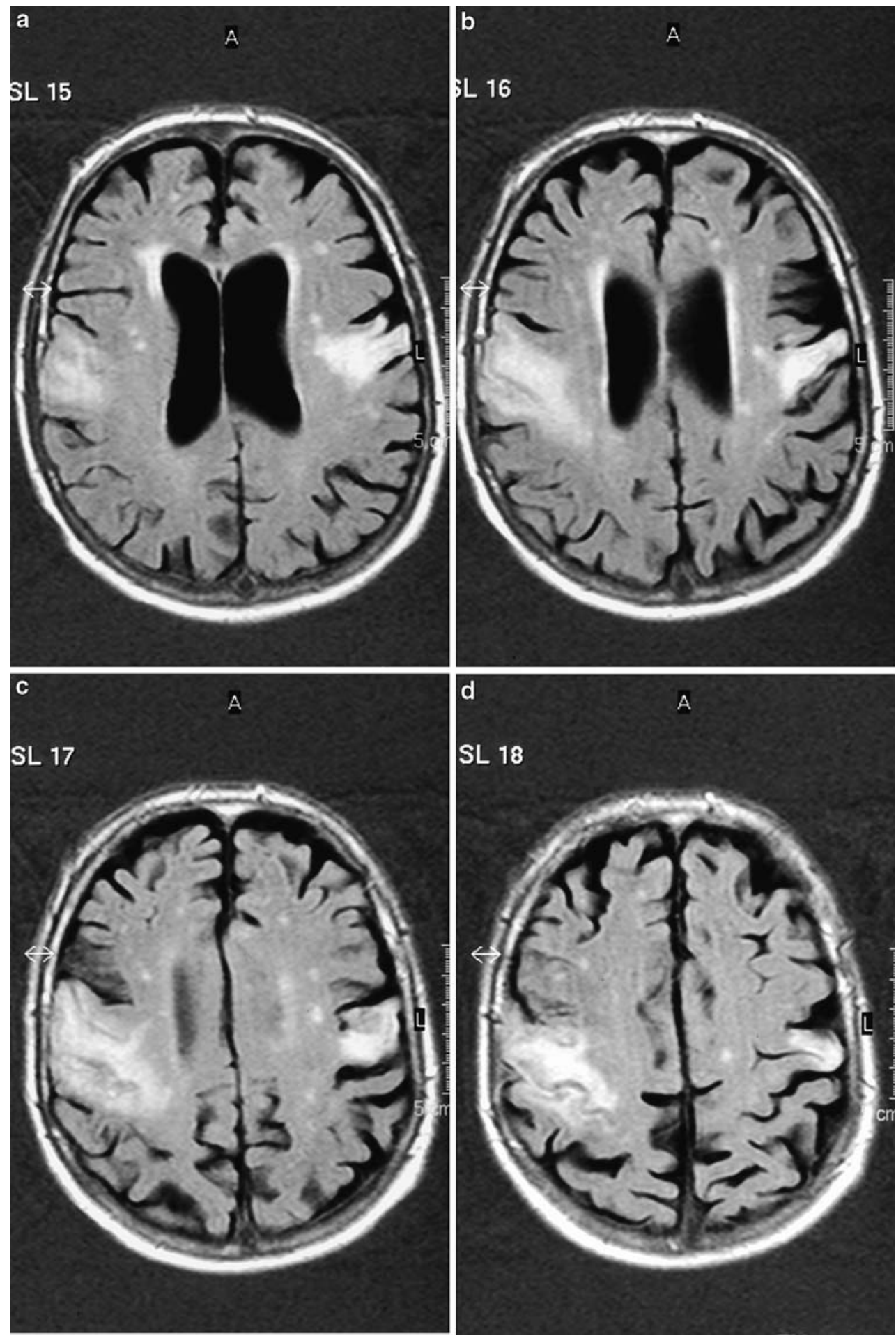

Fig. 1 MRI scan performed after the second stroke. The scan shows diffuse atrophy of the brain, an old left-sided cortical infarction, a new infarction in the right pre- and post-central convolutions, and right-sided subcortical lacunar foci 
feeding. He was referred to the dysphagia clinic and offered neuromuscular electrical stimulation of the muscles involved in the swallowing act simultaneous with logopedic dysphagia treatment.

During 5 months, the patient received treatment sessions of $1 \mathrm{~h}$ each, on five consecutive days per week. The major treatment goals are to improve the hyolaryngeal elevation and to stimulate the sensory input that may facilitate volitional triggering of swallowing in dysphagia [16, 17]. Two dual channel modified hand-held battery-powered electrical stimulators (VitalStim ${ }^{\circledR}$ Therapy; frequency $80 \mathrm{~Hz}$, pulse width $700 \mu$ s, Chattanooga Group, Chattanooga, TN, USA) were used by an experienced speech therapist. The initial intensity of the electrical current was $6.5 \mathrm{~mA}$ in position "A" (Fig. 2a) using two sets of snap skin electrodes, located on each side of the midline, above and beneath the lesser horns of the hyoid bone, on the mylohyoid muscles, and on the thyrohyoid muscles. Position "B" (Fig. 2b) using one set of electrodes, located on each side of the mouth, on the orbicularis oris muscle and the masseter muscles. The highest electrical current level the patient could tolerate, resulting in maximum muscle contraction without spasm, was applied as maximum motor level in position " $A$ " and "B" [17]. During the following sessions, the position $B$ and the combination of position $\mathrm{A}$ with $\mathrm{B}$ were alternatively applied with increasing intensity of the electrical current up to $17.5 \mathrm{~mA}$ for position $\mathrm{B}$ and $10.0 \mathrm{~mA}$ for position A. Logopedic dysphagia treatment was given simultaneously with electrical stimulation. Initial small amounts (2 till 5 $\mathrm{ml}$ ) of thickened liquids were offered to the patient progressively followed by the intake of various food consistencies, using swallowing manoeuvres $[6,15]$.

Before and after treatment, a clinical assessment (Table 1) was performed by the speech therapist using an oral motor function test (5-point scale) and a functional oral intake scale for dysphagia according to Crary [5]. No considerable difference of voluntary control of swallowing was present at the end of therapy as was confirmed by the patient's inability to perform six out of seven voluntary exercises of the oral motor function test. Only minor movements of the lips were observed while the speech therapist was presenting oral movements as imitative tasks. During therapy the functional oral intake scale showed changes from PEG tube feeding to oral diet with multiple consistencies requiring special preparation or compensations [5]. Clinically, the presence of the swallowing reflex could be observed. However, the patient's extreme weak condition and inability to swallow without great risk of massive aspiration, did not allow a pretreatment videofluoroscopic examination (VFS), neither a fiberoptic endoscopic evaluation of swallowing (FEES). After therapy, both assessment techniques were performed successfully. The FEES was performed by the laryngologist using a standardized FEES protocol [14]. The FEES and videofluoroscopy were judged independently by the speech therapist and laryngologist using the "penetration-aspiration scale" according to Rosenbek [21] (Table 1).

During the posttreatment FEES, the patient identified sensory testing correctly. The oral transit was severely insufficient so that the bolus had to be positioned at the base of the tongue by means of a tablespoon to initiate the swallowing reflex. The reflexive phase of swallowing could be observed. The pharyngeal delay time [14] was measured from the moment the bolus was positioned at the base of the tongue until the swallowing reflex was triggered while the endoscopic image was in the "home" position [14]. The pharyngeal delay time was less then $0.40 \mathrm{~s}$ for both thin and thickened liquid boluses of $5 \mathrm{ml}$. After swallowing, mild symmetrical pooling in the pyriform cavities was observed without laryngeal penetration nor aspiration, resulting in a score of one on the "penetration-aspiration scale" [21].

During the first attempt of the posttreatment VFS ( $3 \times 10 \mathrm{ml}$ high-density barium boluses), minor aspiration during swallowing was observed. A strong coughing reflex immediately evacuated this partially aspirated bolus. The response of the patient to aspiration was six on the "penetration-aspiration scale" [21] (Table 1). During the two other swallowing attempts, neither penetration nor aspiration was observed.

In conclusion, posttreatment, dysphagia with minor diet restrictions, consisting of multiple consistencies requiring special preparation, was observed [5]. One year after therapy, the patient's clinical condition has not changed; he is still on oral diet without pneumonia.

\section{Comment}

The primary neural pathway for voluntary swallowing is blocked in the opercular syndrome [1, 13, 22]. The voluntary control of the oral musculature is absent, resulting in disuse of the swallowing musculature, with preservation of the reflexes like the swallowing reflex [22]. In general, disuse of striated muscles leads to atrophy and loss of muscle force $[12,20]$. Electrical stimulation is a well-documented clinical method of augmenting muscle performance in both normal and paralyzed muscles [7, 11, 20]. Some studies report an increase in contractile qualities [19] and muscle force after electrical stimulation [12]. According to Hainaut et al. NMES as a training modality is not a substitute for, but a complement of voluntary exercise of disused and healthy muscles [11]. In this patient, electrical stimulation was applied simultaneous with logopedic dysphagia treatment. Electrical stimulation has allowed restoration of the motor function of paralyzed muscles [12]. It can also initiate muscle re-education $[10,16]$ and may create a relearn- 
Fig. 2 The position of the skin electrodes. a On each side of the midline, above and beneath the lesser horns of the hyoid bone, on the mylohyoid muscles, and on the thyrohyoid muscles. b On each side of the mouth, on the orbicularis oris muscle and the masseter muscles a

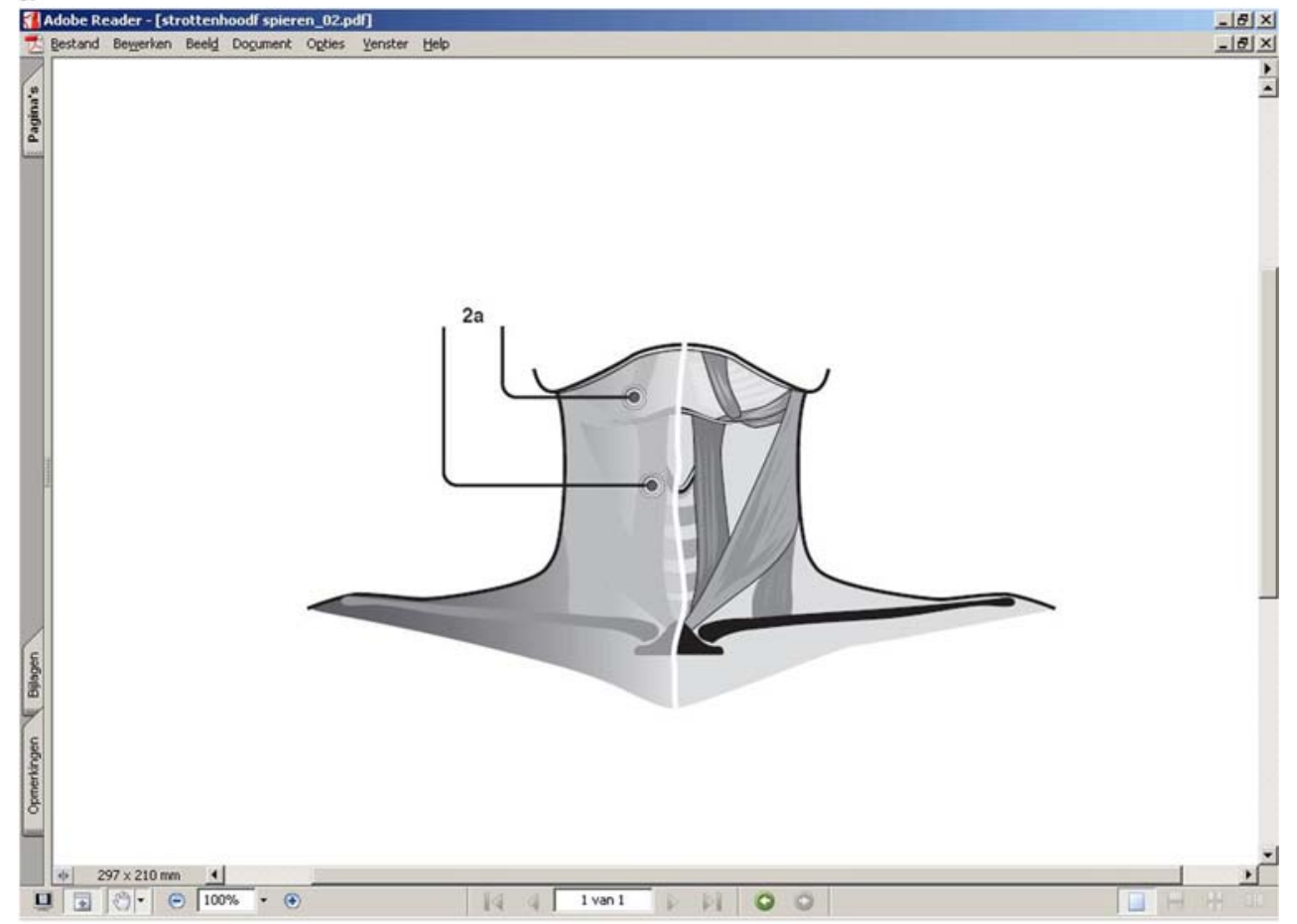

b

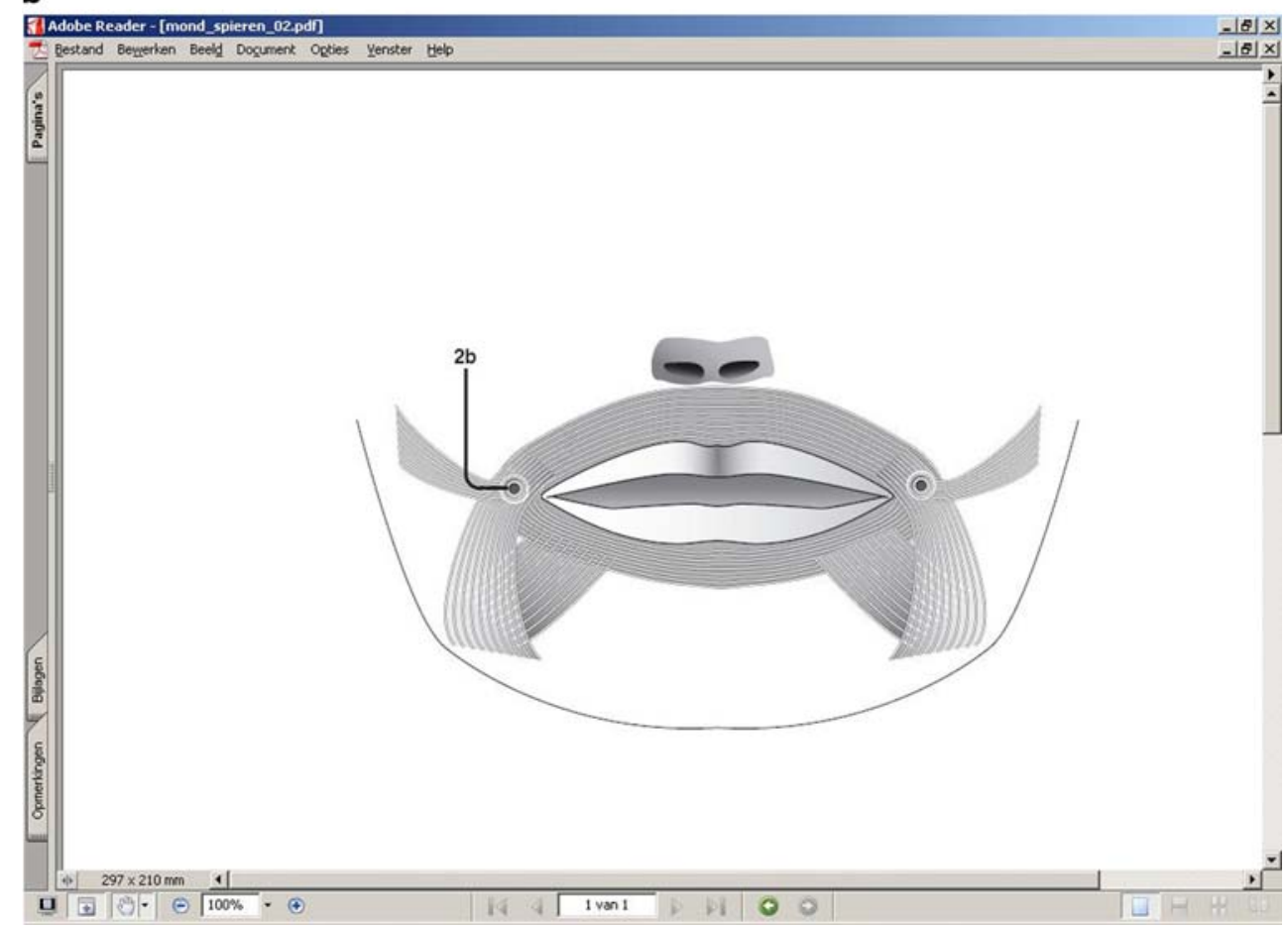

ing process of the brain in controlling the muscles responsible for the swallowing act [16]. Leelamanit et al. found that surface electrical stimulation of the thyrohyoid muscles helps to improve the swallow function by optimized hyolaryngeal elevation [16]. These studies suggest a positive effect with improvement of the swallowing function. Burnett et al. observed that paired intramuscular stim- ulation of the mylohyoid and/or thyrohyoid muscles produced about $50 \%$ of the laryngeal elevation with about $80 \%$ of the velocity that normally occurs during a swallow [3]. Hyolaryngeal elevation plays an important role in the cricopharyngeal opening $[4,16]$. According to Ludlow et al. sensory levels of surface electrical stimulation may facilitate triggering of swallowing in dysphagia [17]. 


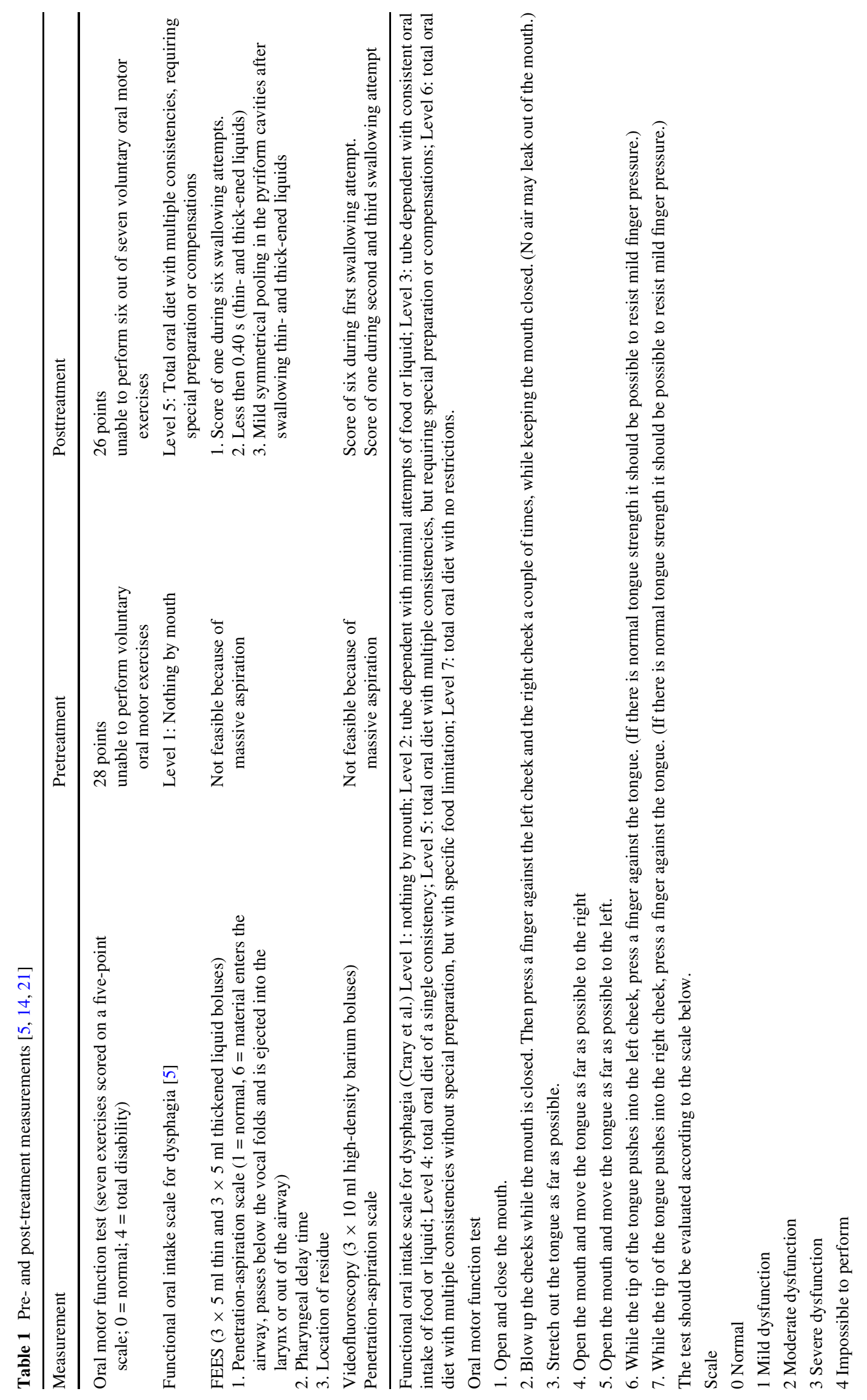


In this patient, the muscles involved in swallowing were presumed to be atrophied because of disuse due to complete loss of voluntary control and PEG tube feeding. It is hypothesized, that in this patient after surface electrical stimulation combined with logopedic dysphagia treatment, an improvement of the reflexive and automatic movements during the swallowing act appeared, based on an increase of contractile qualities and muscle force. Despite the fact that a pretreatment FEES and a videofluoroscopic examination could not be performed in this patient, posttreatment improved muscle force resulting in improvement of amplitude of the hyolaryngeal elevation, and improvement of the cricopharyngeal opening during the reflexive phase of the swallowing act, are presumed. Despite some movements of the lips, the voluntary control of the initiation of the swallowing act did not improve.

A second hypothesis is based on NMES serving as a cue to break through the apraxia [2]. In literature, some patients with opercular syndrome have been described as suffering from buccofacial and/or verbal apraxia, especially those with lesions extending to Broca's area. Non-verbal oral movements, being less complex than articulatory movements, may recover in some cases of oral apraxia [8]. During the treatment consisting of logopedic dysphagia training combined with NMES, the NMES may have served as a proprioceptive cue. This phenomenon could explain some voluntary oral movements in this patient during the application of NMES without imitative tasks at the end of therapy.

No universal accepted protocol for surface electrical stimulation for dysphagia, including intensity of the current, frequency and length of the treatment, does exist at the moment. Neuromuscular electrical stimulation as a treatment for dysphagia has been documented in the literature rarely. Furthermore, dysphagia treatment in the opercular syndrome has never been reported yet. This case report describes the possibility of neuromuscular electrical stimulation to treat dysphagia in the opercular syndrome. Neuromuscular electrical stimulation as a treatment for dysphagia is a promising paramedical technology that has to be studied further to specify the treatment protocol as well as the medical indications.

Open Access This article is distributed under the terms of the Creative Commons Attribution Noncommercial License which permits any noncommercial use, distribution, and reproduction in any medium, provided the original author(s) and source are credited.

\section{References}

1. Bakar M, Kirshner HS, Niaz F (1998) The opercular-subopercular syndrome: four cases with review of the literature. Behav Neurol 11:97-103

2. Borod JC, Lorch MP, Koff E, Nicholas M (1987) Effect of emotional context on bucco-facial apraxia. J Clin Exp Neuropsychol 9:155-161
3. Burnett TA, Mann EA, Cornell SA, Ludlow CL (2003) Laryngeal elevation achieved by neuromuscular stimulation at rest. J Appl Physiol 94:128-134

4. Cook IJ, Dodds WJ, Dantas RO, Massey B, Kern MK, Lang IM, Brasseur JG, Hogan WJ (1989) Opening mechanisms of the human upper esophageal sphincter. Am J Physiol 257:748-759

5. Crary MA, Carnaby Mann GD, Groher ME (2005) Initial psychometric assessment of a functional oral intake scale for dysphagia in stroke patients. Arch Phys Med Rehabil 86:1516-1520

6. Crary MA, Groher ME (2003) Overview of behavioral treatment strategies. In: Crary MA, Groher ME (eds) Introduction to adult swallowing disorders. Butterworth Heinemann, St. Louis, pp 179 198

7. Delitto A, Snyder-Mackler L (1990) Two theories of muscle strength augmentation using percutaneous electrical stimulation. Phys Ther 70:158-164

8. De Renzi E, Pieczuro A, Vignolo LA (1966) Oral apraxia and aphasia. Cortex 2:50-73

9. Foix C, Chavany JA, Marie J (1926) Diplegie facio-linguo-masticatrice d'origine cortico-sous-cortical sans paralysie des membres. Rev Neurol 33:214-219

10. Freed M, Freed L, Chatburn R, Christian M (2001) Electrical stimulation for swallowing disorders caused by stroke. Respir Care 46:466-474

11. Hainaut K, Duchateau J (1992) Neuromuscular electrical stimulation and voluntary exercise. Sports Med 14:100-113

12. Kagaya H, Shimada Y, Sato K, Sato M (1996) Changes in muscle force following therapeutic electrical stimulation in patients with complete paraplegia. Paraplegia 34:24-29

13. Lang C, Reichwein J, Iro H, Treig T (1989) Foix-Chavany-Marie syndrome-neurological, neuropsychological, CT, MRI, and SPECT findings in a case progressive for more than 10 years. Eur Arch Psychiatr Neurol Sci 239:188-193

14. Langmore SE, Aviv JE (2001) Endoscopic procedures to evaluate oropharyngeal swallowing. In: Langmore SE (ed) Endoscopic evaluation and treatment of swallowing disorders. Thieme, New York, pp 73-100

15. Langmore S, Miller RM (1994) Behavioral treatment for adults with oropharyngeal dysphagia. Arch Phys Med Rehabil 75:1154 1159

16. Leelamanit V, Limsakul C, Geater A (2002) Synchronized electrical stimulation in treating pharyngeal dysphagia. Laryngoscope 112:2204-2210

17. Ludlow CL, Humbert I, Saxon K, Poletto C, Sonies B, Crujido L (2007) Effects of surface electrical stimulation both at rest and during swallowing in chronic pharyngeal dysphagia. Dysphagia 22:1-10

18. Magnus A (1837) Fall von Aufhebung des Willenseinflusses auf einige Hirnnerven. Müllers Arch Anat Physiol Wissensch Med 258-266

19. Martin L, Cometti G, Pousson M, Morlon B (1993) Effect of electrical stimulation training on the contractile characteristics of the triceps surae muscle. Eur J Appl Physiol 67:457-461

20. Merletti R, Zelaschi F, Latella D, Galli M, Angeli S, Bellucci Sessa M (1987) A control study of muscle force recovery in hemiparetic patients during treatment with functional electrical stimulation. Scand J Rehabil Med 10:147-154

21. Rosenbek JC, Robbins JA, Roecker EB, Coyle JL, Wood JL (1996) A penetration-aspiration scale. Dysphagia 11:93-98

22. Weller M (1993) Anterior opercular cortex lesions cause dissociated lower cranial nerve palsies and anarthria but no aphasia: FoixChavany-Marie syndrome and "automatic voluntary dissociation" revisited. J Neurol 240:199-208

23. Weller M, Poremba M, Dichgans J (1990) Opercular syndrome without opercular lesions: Foix-Chavany-Marie syndrome in progressive supranuclear motor system degeneration. Eur Arch Psychiatr Neurol Sci 239:370-372 